\title{
Effective Trilateration-based Indoor Localization Method Utilizing Active Control of Lighting Devices
}

\author{
Kazuki Moriya, ${ }^{1}$ Manato Fujimoto, ${ }^{1 *}$ Yutaka Arakawa, ${ }^{2}$ and Keiichi Yasumoto ${ }^{1}$ \\ ${ }^{1}$ Graduate School of Science and Technology, Nara Institute of Science and Technology, \\ 8916-5 Takayama-cho, Ikoma, Nara 630-0192, Japan \\ ${ }^{2}$ Department of Advanced Information Technology, Kyushu University, \\ 744 Motooka, Nishi-ku, Fukuoka, Fukuoka 819-0395, Japan
}

(Received September 11, 2019; accepted January 9, 2020)

Keywords: indoor localization, trilateration method, active control, distance-illuminance model, lighting device

In this paper, we propose a novel trilateration-based indoor localization method utilizing active control of lighting devices. The proposed method estimates the position of a target for measurement by utilizing a trilateration method based on the distances between the target and lighting devices calculated from the illuminance value obtained by active control. The active control involves turning on lighting devices newly installed in the target area one by one. Our method has three key ideas. The first is to remove the effect of light sources other than the lighting equipment used for localization by utilizing only the difference between the illuminance values obtained before and after turning on each lighting device. The second is to remove the influence of a human shadow by utilizing three lighting devices used for localization selected from four lighting devices newly installed in the target area. The third is to accurately estimate the position of a target by introducing a distance-illuminance model that can calculate the distance between an illuminance sensor and a lighting device accurately. To show the effectiveness of the proposed method, we conducted evaluation experiments. It was found that the proposed method can estimate the position of a target with approximately $1 \mathrm{~m}$ error on average.

\section{Introduction}

Recently, owing to the astonishing progress of ubiquitous computing technology, various applications utilizing indoor location-based services (LBSs) have been developed. ${ }^{(1,2)}$ In particular, these days, smart home applications such as home appliance control to reduce energy consumption, ${ }^{(3,4)}$ concierge services for residents, ${ }^{(5)}$ and monitoring systems for elderly people ${ }^{(6,7)}$ are attracting attention. To realize these LBS applications, it is necessary to recognize a wide variety of daily living activities of people with high accuracy and low cost. Therefore, studies on activity recognition utilizing indoor location information have been conducted actively in recent years. ${ }^{(8,9)}$ 
As a representative example of an activity recognition study, Ueda et al. ${ }^{(10)}$ have proposed an effective method for accurately recognizing 10 types of daily living activity, such as cooking, watching TV, and taking a bath, by utilizing information of indoor locations and the power consumption of home appliances. In this work, an average estimation accuracy of approximately $87.1 \%$ as well as a localization accuracy within $1.4 \mathrm{~m}$, was achieved by utilizing three power meters. From this result, we found that realizing an effective indoor localization method that can achieve a localization accuracy of approximately $1 \mathrm{~m}$ is required for more accurate activity recognition.

Several localization methods have been proposed. As a typical localization method, the use of camera-based systems is one of the most well-known methods. Basically, multiple cameras are installed in a room, and they estimate the positions of people/objects by image processing. ${ }^{(1,12)}$ However, since these methods require the installation of many cameras, they are costly. Also, personal privacy is violated as the camera resolution increases.

Passive radio frequency identification (RFID)-based localization methods have also been proposed. These methods mainly estimate the positions of people/objects utilizing the directivity formed by the combination of a reader antenna and an RFID tag. ${ }^{(13,14)}$ However, these methods require one or more readers to receive radio waves from the RFID tag. Thus, the deployment cost is very high for an ordinary user.

Wi-Fi/BLE signal-based localization methods have also been proposed. ${ }^{(15-20)}$ These methods realize localization to some level of accuracy by employing trilateration, fingerprinting, signal strength (SS), time difference of arrival (TDoA), time of arrival (ToA), and angle of arrival (AoA). However, the localization accuracy is significantly reduced in some cases (e.g., to approximately 5 to $10 \mathrm{~m}$ ) owing to the reflection and diffraction of signals since the Wi-Fi/BLE signal is very unstable.

Currently, a localization method utilizing visible light (VL) has attracted attention as one of the methods that can solve the problems of existing localization methods. ${ }^{(21,22)}$ The VL-based localization method estimates the positions of people/objects with high accuracy by utilizing the difference in light intensity obtained by an illuminance sensor installed in an indoor environment. Since this method uses only an illuminance sensor and lighting devices for localization, the installation cost is low and user privacy is not violated. However, this method has the following two serious problems. The first problem is that the method cannot maintain sufficient localization accuracy when it is affected by light sources other than the lighting devices used for localization, such as sunlight. The second problem is that it cannot estimate the position of a user accurately owing to the influence of the human shadow when the user wears a wearable illuminance sensor such as glasses.

In this paper, we propose a novel trilateration-based indoor localization method utilizing an active control of lighting devices to solve the problems of the VL-based localization method. The active control involves turning on lighting devices newly installed in the target area one by one. Specifically, the proposed method first turns on three (or four) lighting devices newly installed in the target area one by one. The illuminance value at the target observation point is measured by an illuminance sensor. Then, the proposed method calculates the distance between the illuminance sensor and each lighting device by applying the measured illuminance value to 
obtain a distance-illuminance model. This model gives the relationship between the distance between the illuminance sensor, which is the target for measurement, and a lighting device and the illuminance value. Finally, the proposed method estimates the position of the illuminance sensor by utilizing the trilateration method based on the calculated distance information. We have already proposed the basic idea of this method in our previous work. ${ }^{(23)}$ However, we have not solved all the problems of the VL-based localization method. In this paper, we extend the previous work to solve the problems. Specifically, the main contributions of this paper are summarized as follows:

- First, we propose an algorithm that can remove the effect of light sources other than the lighting devices used for localization (e.g., sunlight and ceiling light) by utilizing only the difference between illuminance values obtained before and after turning on each lighting device.

- Second, as an extension of the method in our previous work, ${ }^{(23)}$ we propose a novel algorithm that can remove the influence of a human shadow by utilizing three lighting devices used for localization selected from four lighting devices newly installed in the target area.

- Finally, we show that the proposed method can accurately estimate the position of a target for measurement by introducing a distance-illuminance model, which can calculate the distance between an illuminance sensor and a lighting device accurately.

The rest of this paper is organized as follows. Section 2 reviews the existing work related to this paper. Section 3 gives a definition of the problem in our study and Sect. 4 presents our proposed trilateration-based indoor localization method. The preliminary experiment is described in Sect. 5 and the evaluation experiments are described in Sect. 6. Finally, Sect. 7 concludes this paper.

\section{Related Work}

Many indoor localization methods related to our research that employ several techniques, such as Wi-Fi, BLE, magnetic fields, RFID, infrared, and ultrasounds, have been proposed. ${ }^{(24,25)}$

As a typical localization method, there are several studies in which the position of the user was estimated by utilizing stereo cameras. Krumm et al. ${ }^{(11)}$ have proposed a vision system that uses several stereo cameras to track multiple people simultaneously. Checka et al. ${ }^{(12)}$ have proposed an effective multimodal tracking framework that can track multiple people by using a combination of audio and video observations. These systems have several stereo cameras installed in a room and they estimate the position of the user by processing the images taken from the cameras. However, to realize indoor localization for every room, the installation of many stereo cameras is required and the cost becomes a burden to the user. Moreover, a stereo camera always captures the posture of the user. Since this intrudes on the user's privacy, these systems are not suitable for our study.

There are several studies of indoor localization that utilize pedestrian dead reckoning (PDR). ${ }^{(26)}$ In these studies, the position of the user is estimated by utilizing inertial sensors, such as an accelerometer and a gyroscope, in a smartphone. However, the reduction in the accumulated 
error of the sensors is challenging. Moreover, Active Bat utilizes ultrasonic waves to estimate the position of a user. ${ }^{(27)}$ This system can estimate the position of the user accurately by utilizing the TDoA technique. However, the ultrasonic localization system costs more than $€ 3000$, which is too high for ordinary users. Therefore, it is difficult to use this system in our target environment.

There are also studies in which the position of the user is estimated by utilizing the transmission of a radio wave. Pilot is a device-free localization system that uses the channel state information (CSI) of a wireless local area network (WLAN). ${ }^{(28)}$ However, this system requires the installation of several access points (APs). Also, a complex site survey of the radio wave is required to use the system. Since the installation and survey cost is a burden on ordinary users, the construction of localization systems using the transmission of a radio wave is very difficult. Therefore, a localization system that can be constructed easily and cheaply is required.

Some approaches utilizing Wi-Fi/BLE signals have also been proposed. These approaches realize localization to some level of accuracy by employing trilateration, ${ }^{(15)}$ fingerprinting, ${ }^{(16)}$ SS, ${ }^{(17)}$ TDoA, ${ }^{(18)}$ ToA, ${ }^{(19)}$ and AoA. ${ }^{(20)}$ However, because the signals of Wi-Fi/BLE are very unstable, the localization accuracy is significantly reduced in some cases (i.e., to approximately 5 to $10 \mathrm{~m}$ ) owing to the reflection and diffraction of signals. Thus, these approaches may not be able to achieve the localization accuracy we are aiming for.

As another approach, a system using the vibration generated from footsteps during a living activity has been proposed. For example, Kashimoto et al. ${ }^{(29)}$ have proposed a piezo sensor-based indoor localization system that estimates the position of a user by utilizing a piezo component attached to the floor. This system estimates the position of the user from the vibration type associated with four living activities (i.e., step, open door, close door, and so forth.) obtained by multiple piezo sensors. Although a system prototype has already been implemented, the localization accuracy obtained using the system has not yet been investigated. Hence, its feasibility is not known.

Several approaches based on a passive RFID system have also been proposed. As one of the representative techniques, Hähnel et al. ${ }^{(13)}$ have proposed an indoor localization method using a probabilistic model called the sensor model, formed by the combination of a reader and an RFID tag. Fujimoto et al. ${ }^{(14)}$ have proposed an indoor localization method that can quickly and accurately estimate the position of an RFID tag simply by rotating a reader so that it faces an observation point. This method estimates the position of an RFID tag attached to an object accurately by utilizing a communication area model that is generated from the communication range corresponding to the relative angle of the reader antenna and RFID tag. Jiang et al. ${ }^{(30)}$ have proposed a method called 3D-OmniTrack, which can accurately track the 3D localization and orientation of a target object. However, in these methods, the installation cost of the passive RFID system is very high since these systems require one or more readers (or special wearable readers/sensors) to receive the radio wave from the RFID tag. Therefore, a low-cost system is required for the target environment.

The approach of utilizing VL is currently attracting attention as a technique that can achieve high position estimation accuracy. ${ }^{(21,22)}$ The VL-based approach basically estimates the 
position of a user and/or object from the difference in light intensity (i.e., illuminance value) obtained by an illuminance sensor installed in an indoor environment. Since this approach uses only an illuminance sensor and lighting devices (i.e., no other special devices are required) for localization, it is a comparatively low-cost and easy-to-construct localization system. Also, this approach can estimate the user's position without accumulating localization errors or infringing on user privacy since it does not use an accelerometer, cameras, or audio. However, this method has two problems. The first is that it cannot maintain sufficient localization accuracy because it is affected by light sources other than the lighting equipment used for localization, such as sunlight. The second is that it cannot estimate a user's position accurately owing to the influence of the human shadow when a user wears a wearable illuminance sensor such as glasses. These problems are important challenges to be solved in our study.

\section{Problem Definition for VL-based Localization}

In this paper, we focus on the VL-based localization method. In this section, we define the problem of the VL-based localization targeted in this paper before describing our proposed method. First, we describe the prerequisites of the target space and lighting devices used for our study. Then, we define the problem of the VL-based localization in our study. Finally, we describe the approach used to solve the problems in our study.

\subsection{Prerequisites of target space}

We first describe the prerequisites of the target space in this study. We assume an ordinary room in a general house as the target space. Figure 1 shows an example of a target space. As shown in Fig. 1, we assume that the area (i.e., the area in pink) that is affected by newly installed lighting devices at the edge of the target space is the target area in which localization is conducted. We assume that the following prerequisites are satisfied in the target space:

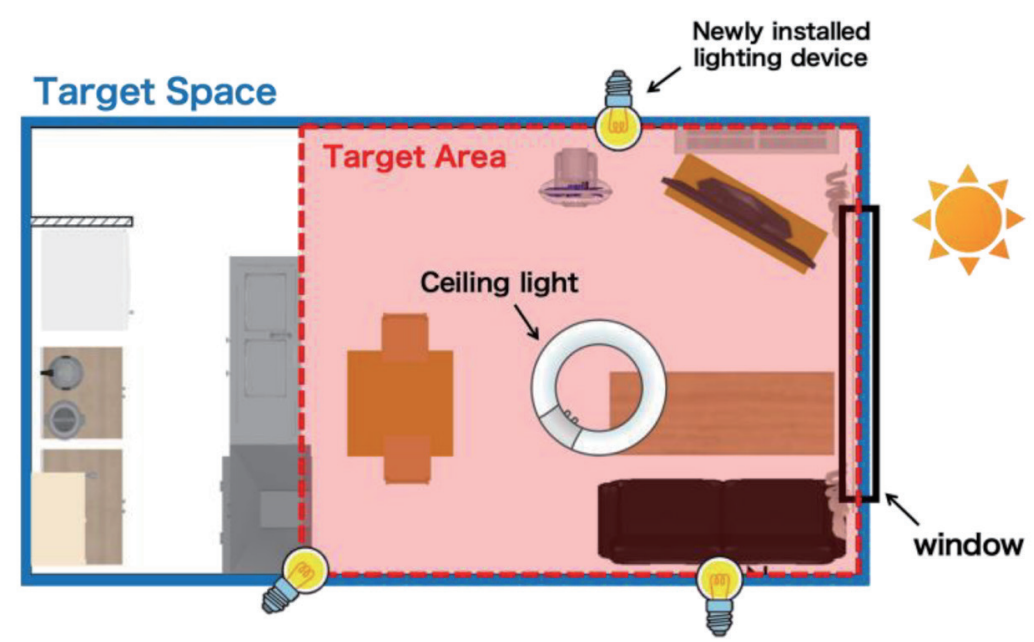

Fig. 1. (Color online) Example of target space. 
- Multiple lighting devices can be newly installed at the edge of the target area.

- Ordinary furniture, home appliances, and ceiling lights are installed in the target area.

- Sunlight enters the target area from a window installed in the target space.

\subsection{Prerequisites of lighting devices}

Next, we explain the prerequisites of the lighting devices used in this study. The VC-based localization method estimates the position of a target for measurement from the difference in illuminance value obtained by an illuminance sensor. Thus, this method does not use accelerometers, cameras, or voice to avoid an accumulated error and protect the privacy of users. Also, since this method uses multiple lighting devices to conduct localization, it has to construct a localization system by utilizing devices that can be installed at a comparatively low cost. Therefore, we consider that it is necessary to use lighting devices that are widely used in general households to easily construct a localization system. Additionally, it is necessary to be able to easily switch the state of lighting devices (i.e., ON and OFF) at any time to measure the difference in light intensity. Hence, the prerequisites of the lighting devices used in this study are summarized as follows:

- Incandescent or LED lights used as ordinary lighting devices are used for localization.

- There are two states of the lighting devices: ON and OFF.

- The state of the lighting devices can be changed by remote control at any time.

- The coordinates of each lighting device installed for localization are known.

As the target for measurement in this paper, we assume that an illuminance sensor can be placed at any position in a target area or that a user wears a wearable illuminance sensor.

\subsection{Problem definition}

In this section, we define the problem. Figure 2 shows a conceptual diagram of the problem definition for VC-based localization. The set of target areas in the target space that satisfy the prerequisites mentioned in Sect. 3.1 is denoted by $A$. The set of lighting devices (indicated by circles) newly installed at the edge of each target area $a(a \in A)$ is denoted by $L=\left\{l_{1}, l_{2}, \cdots, l_{n}\right\}$, and the set of ceiling lights is denoted by $L^{\prime}=\left\{l_{1}^{\prime}, l_{2}^{\prime}, \cdots, l_{n}^{\prime}\right\}$. The position coordinate of an illuminance sensor, which is the target for measurement (indicated by a star), installed at an

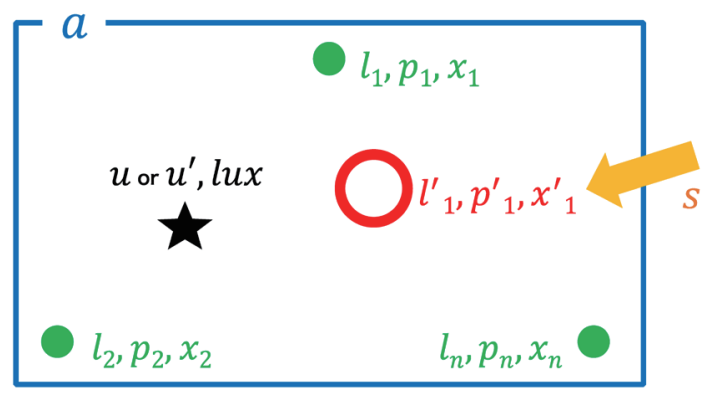

Fig. 2. (Color online) Diagram of problem definition. 
arbitrary position in $a$ is denoted by $u$, or the position coordinate of a user wearing a wearable illuminance sensor is denoted by $u^{\prime}$. The illuminance value measured by an illuminance sensor is denoted by lux. The state of the lighting device $l_{i}\left(l_{i} \in L\right)$ is denoted by $p_{i}(\mathrm{ON} / \mathrm{OFF})$ and the installation coordinate is denoted by $x_{i}$. The sets of $p_{i}$ and $x_{i}$ are denoted by $P=\left\{p_{1}, p_{2}, \cdots, p_{n}\right\}$ and $X=\left\{x_{1}, x_{2}, \cdots, x_{n}\right\}$, respectively. $p_{i}^{\prime}, x_{i}^{\prime}, P^{\prime}$, and $X^{\prime}$ are similarly defined for a ceiling light (indicated by a ring). Also, the state (with/without) of sunlight (indicated by an arrow) entering area $a$ is denoted by $s$. The aim of our study is to estimate $u$ or $u^{\prime}$ when lux, $L, P$, and $X$ are given in an arbitrary $a \in A$.

\section{Proposed Method}

In this section, we describe our proposed VL-based localization method. First, we give an outline of the proposed method. Then, we describe the localization algorithms of the proposed method. Finally, we describe the distance-illuminance model, which is the core mechanism in our proposed method.

\subsection{Outline}

The ultimate goals of this paper are (1) to propose a novel localization method that can solve the problems of the VL-based approach and (2) to investigate the effectiveness of the proposed method by performing evaluation experiments assuming a real environment. To achieve these objectives, we found that our proposed method needs to fulfill the following three main requirements as discussed in Sects. 2 and 3:

Req. 1: The method should be able to estimate the target's position even when there is an influence of external light, such as sunlight.

Req. 2: The method should be able to estimate the target's position even in an environment influenced by a human shadow.

Req. 3: The method should be able to achieve sufficient localization accuracy (specifically, approximately $1 \mathrm{~m}$ ).

In this paper, to realize an effective approach that can fulfill all the above requirements while making the best use of the advantages of VL-based localization, we propose a novel trilateration-based indoor localization method utilizing active control of lighting devices. The active control involves turning on the lighting devices newly installed in the target area one by one. The proposed method estimates the position of the target for measurement (illuminance sensor) by utilizing a trilateration method based on the distances between the target for measurement and lighting devices calculated from the difference in illuminance value obtained by active control.

There are three key ideas of the proposed method in order to fulfill Reqs. 1 to 3 . The first idea is that the proposed method can ignore the effect of light sources other than the lighting equipment used for localization (e.g., sunlight and ceiling light) by utilizing only the difference between illuminance values obtained before and after turning on each lighting device (Req. 1). The second idea is that the proposed method can ignore the influence of a human shadow by 
utilizing three lighting devices used for localization selected from four lighting devices newly installed in the target area (Req. 2). The third idea is that the proposed method can accurately estimate the position of a target for measurement by introducing a distance-illuminance model that can calculate the distance between an illuminance sensor and a lighting device accurately (Req. 3). Also, the proposed method can estimate the position of a target for measurement accurately not only in a 2D environment but also in a 3D environment.

\subsection{Localization algorithms}

In this section, we describe the following two localization algorithms of the proposed method: Algorithm 1 is a localization algorithm used to estimate the position of the illuminance sensor and Algorithm 2 is a localization algorithm used to estimate the position of a user who is wearing a wearable illuminance sensor. Before describing the proposed localization algorithms, we first describe some functions defined uniquely in order to understand them:

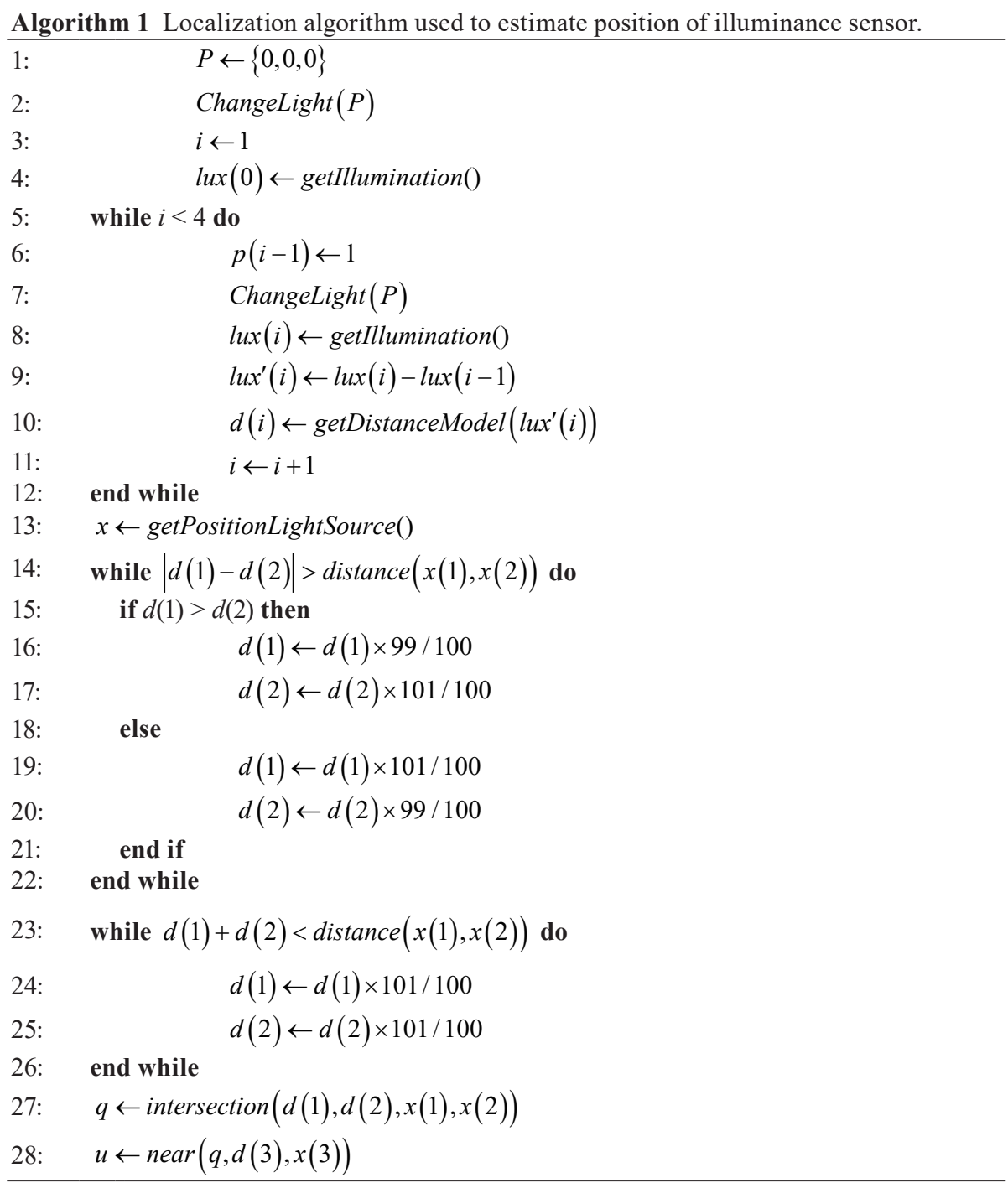




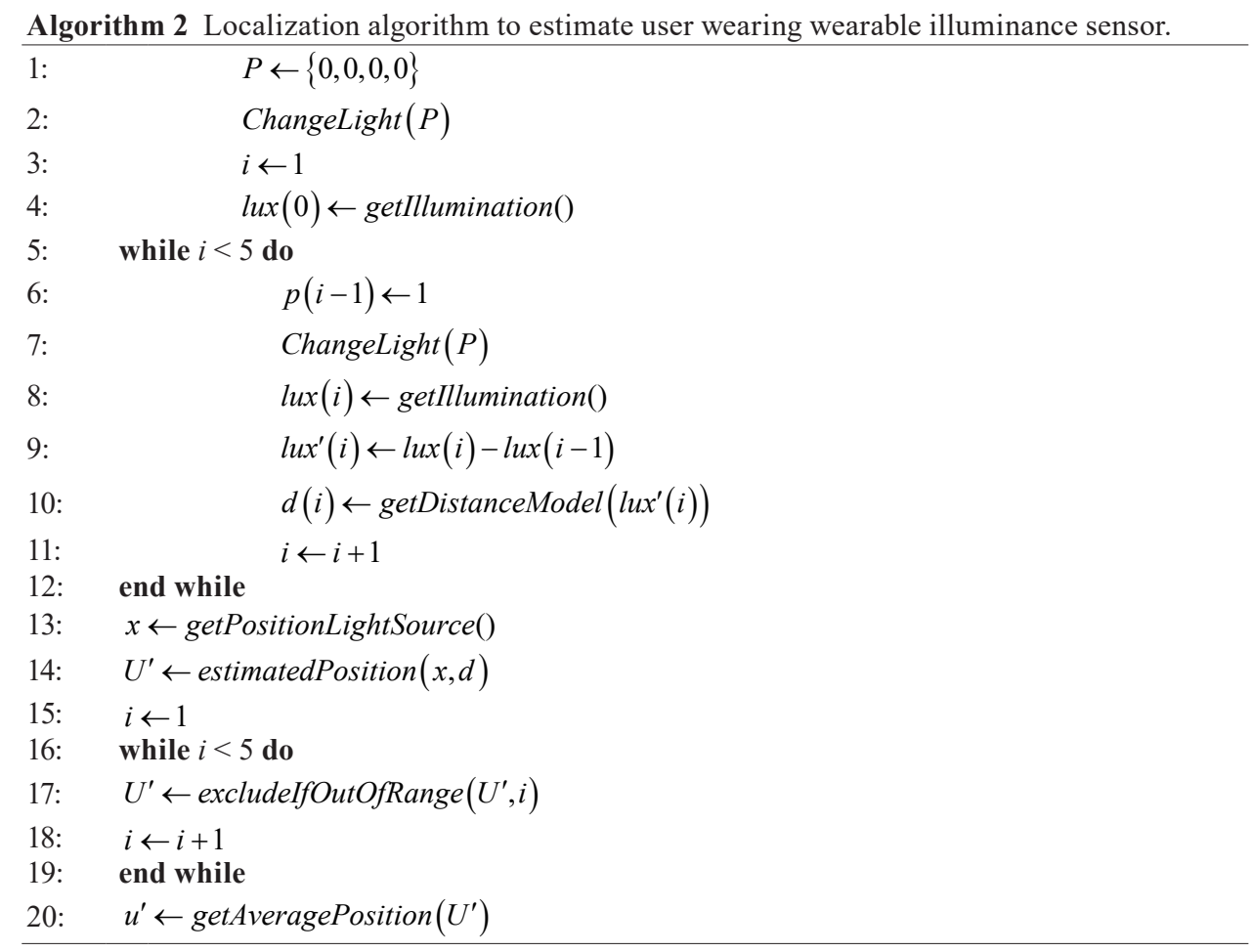

\section{- ChangeLight $(P)$}

Change each lighting device to the lighting state corresponding to pattern $P$.

- getIllumination()

Get the illuminance value measured by the illuminance sensor at the moment.

- $\quad$ getDistanceModel $\left(\operatorname{lux} x^{\prime}(i)\right)$

Get the distance between a lighting device and an illuminance sensor utilizing the distance-illuminance model with the measured illuminance value as the argument.

- getPositionLightSource()

Get the installation coordinates of three lighting devices.

- $\operatorname{distance}\left(x_{1}, x_{2}\right)$

Get the distance between two coordinates $x_{1}$ and $x_{2}$.

- intersection $\left(d_{1}, d_{2}, x_{1}, x_{2}\right)$

Get the intersections of two circles/spheres with center $x$ and radius $d$.

- $\quad n e a r(q, d, x)$

Get the intersection closer to the circle/sphere represented by $x$ and $d$ among $q$ values, which indicate intersection coordinates.

- estimatedPosition $(x, d)$

Get each estimated position coordinate of the four combination patterns described in Sect. 4.2.2 using $x$ and $d$. 
- $\quad$ excludeIfOutOfRange $\left(U^{\prime}, i\right)$

Exclude $U^{\prime}(i)$ from $U^{\prime}$ if $U^{\prime}(i)$ is a position coordinate outside of the target area.

- $\quad$ getAveragePosition $\left(U^{\prime}\right)$

Get the average of all position coordinates of $U^{\prime}$.

In the above, $P$ indicates the set of lighting patterns of each lighting device. For example, when the state of the third lighting device is ON and that of the other lighting devices is OFF, $P$ is denoted by $\{0,0,1\}$ or $\{0,0,1,0\}$. lux $x^{\prime}(i)$ indicates the illuminance value considering only the influence of the $i$ th lighting device, $x$ indicates the installation coordinate of the lighting device, $d$ indicates the distance between the illuminance sensor and the lighting device, $q$ indicates the intersection coordinates obtained from intersection(), and $U^{\prime}$ indicates the set of $u^{\prime}$. Also, the proposed method is trilateration-based indoor localization. Therefore, our algorithms use the intersection of three circles as the estimated position in the case of 2D localization and the intersection of three spheres as the estimated position in the case of 3D localization. In the following sections, we describe the localization algorithms of the proposed method.

\subsubsection{Localization algorithm used to estimate position of illuminance sensor (Algorithm 1)}

First, we describe the localization algorithm used to estimate the position of an illuminance sensor. Basically, when the proposed method estimates the position of the illuminance sensor installed in target area $a$ (hereafter, called $u$ ), it estimates $u$ by using three lighting devices $L=\left\{l_{1}, l_{2}, l_{3}\right\}$ newly installed in a target space. The distance $D=\left\{d_{1}, d_{2}, d_{3}\right\}$ between each lighting device and an illuminance sensor can be estimated by applying the difference between illuminance values observed before and after turning on each lighting device to a distance-illuminance model based on the results of preliminary experiments (refer to Sect. 4.3). The key idea of this algorithm is that it can ignore the effect of light sources other than the lighting devices used for localization (e.g., sunlight and ceiling light) by considering only the difference between illuminance values obtained before and after turning on each lighting device (corresponding to Req. 1). The installation coordinate $X=\left\{x_{1}, x_{2}, x_{3}\right\}$ of each lighting device is already known, as described in Sect. 3.1. Hence, as shown in Fig. 3, this algorithm can estimate $u$ by applying the installation coordinate $X$ and distance $D$ of each lighting device to the trilateration method. The details of this algorithm are described in Algorithm 1 and summarized as follows:

1. First, the state of all lighting devices used for localization starts from OFF (called the initial state). The algorithm first obtains the illuminance value in the initial state (lines $1-4)$.

2. After obtaining the illuminance value in the initial state, the algorithm turns on each lighting device alternately (e.g., $l_{1} \rightarrow l_{2} \rightarrow l_{3}$ ) by utilizing the active control. The algorithm calculates the difference between illuminance values obtained before and after turning on a lighting device and obtains the illuminance value after removing the effect of light sources other than the lighting devices used for localization (lines 6-9). 


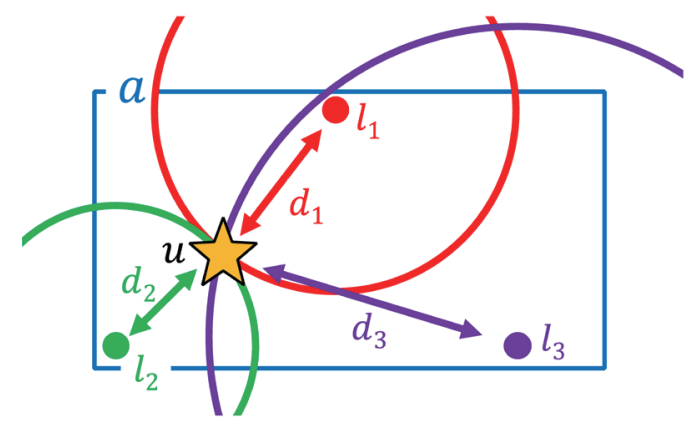

Fig. 3. (Color online) Example of localization to estimate position of illuminance sensor.

3. The algorithm calculates the distance between the target for measurement and a lighting device by applying the illuminance value obtained by the active control to a distanceilluminance model (line 10).

4. The algorithm repeats procedures 2 and 3 until all distances are calculated (lines 5-11).

5. Then, the algorithm creates three circles/spheres with the installation position of each lighting device as the center and with each calculated distance as the radius. After that, the algorithm checks that two circles/spheres selected randomly from the three created circles/spheres intersect (lines 14 and 23).

6. At this time, two cases arise where two circles/spheres do not intersect. The first is the case where both circles/spheres are small, and the second is the case where one circle/sphere is too large compared with the other circle/sphere. If these cases occur, the algorithm repeatedly makes adjustments until the two circles/spheres intersect by changing their size by $1 \%$ intervals (lines $15-21,24$, and 25 ).

7. After the algorithm obtains the two intersecting positions of two circles/spheres, it selects the position closer to the remaining circle/sphere as the final estimated position for a target for measurement (lines 27 and 28).

In the trilateration-based indoor localization method, there are cases where intersections created by three circles/spheres often cannot be calculated due to various factors. In such a case, there is a problem that the existing methods of using the average position of three intersections as the estimated position of the target for measurement cannot be applied. In order to solve such a problem, Paterna et al. ${ }^{(15)}$ have proposed the weighted trilateration method. In our algorithm, based on a part of this method, we adopt a heuristic approach in that two intersections are forcibly created by adjusting (enlarging/reducing) the size of two randomly selected circles/spheres and selecting the intersection closer to the remaining circle/sphere among those intersections as the estimated position of target for measurement.

\subsubsection{Localization algorithm used to estimate position of user wearing wearable illuminance sensor (Algorithm 2)}

Second, we describe a localization algorithm used to estimate the position of a user wearing a wearable illuminance sensor (hereafter, called $u^{\prime}$ ). If a user is wearing a wearable illuminance sensor such as glasses, the illuminance value measured by an illuminance sensor may be 
relatively small since the light emitted from a lighting device is blocked by the user's body (called the influence of the human shadow). Therefore, Algorithm 1 cannot estimate $u^{\prime}$ accurately because it incorrectly calculates the distance between a user and the lighting device to be farther than the actual distance owing to the influence of the human shadow. This algorithm estimates $u^{\prime}$ by utilizing four lighting devices $L=\left\{l_{1}, l_{2}, l_{3}, l_{4}\right\}$ newly installed in the target space to solve this problem. The key idea in this algorithm is that it can ignore the influence of the human shadow by effectively utilizing three lighting devices used for localization selected from four lighting devices by the active control (corresponding to Req. 2). This algorithm uses four patterns corresponding to the combinations $\left(\left\{l_{1}, l_{2}, l_{3}\right\},\left\{l_{1}, l_{2}, l_{4}\right\},\left\{l_{2}, l_{3}, l_{4}\right\}\right.$, and $\left.\left\{l_{1}, l_{3}, l_{4}\right\}\right)$ of the three lighting devices used for localization. For example, as shown in Fig. 4, the algorithm calculates the estimated positions $U^{\prime}=\left\{u_{1}^{\prime}, u_{2}^{\prime}, u_{3}^{\prime}, u_{4}^{\prime}\right\}$ utilizing these patterns by the same procedure as Algorithm 1. The algorithm accurately estimates $u^{\prime}$, which is the final estimated position, by excluding all estimated positions influenced by a human shadow (i.e., estimated

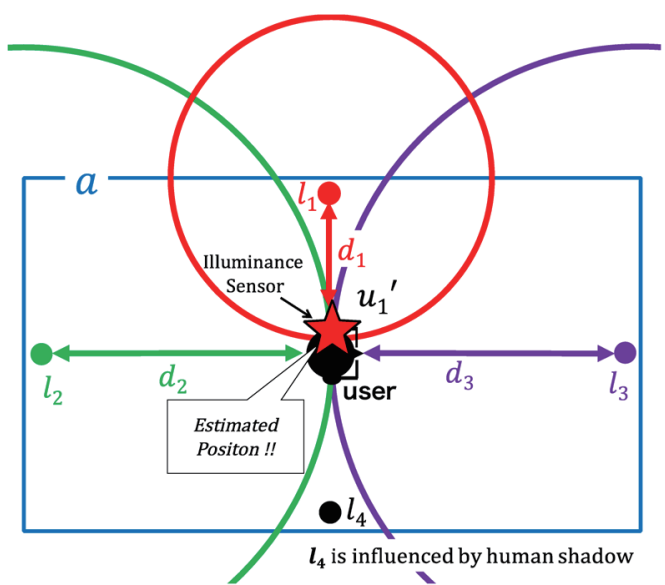

(a)

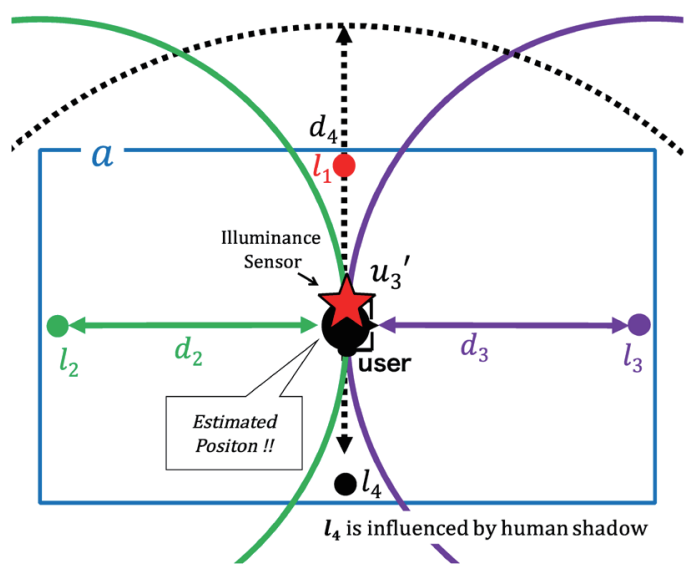

(c)

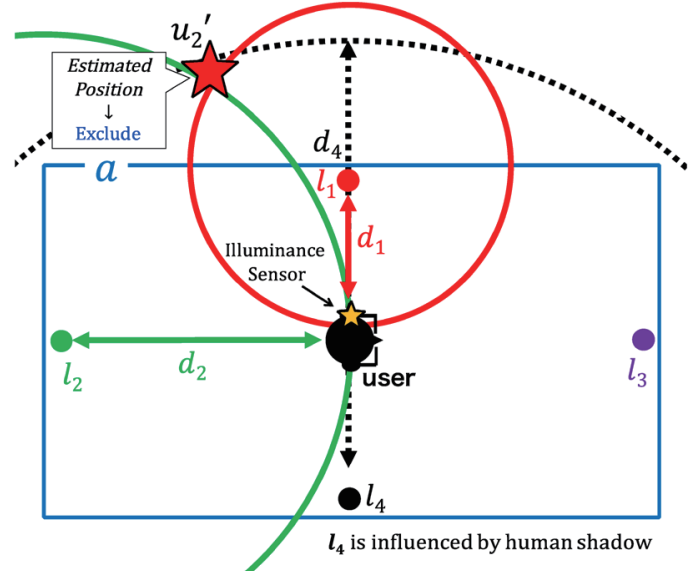

(b)

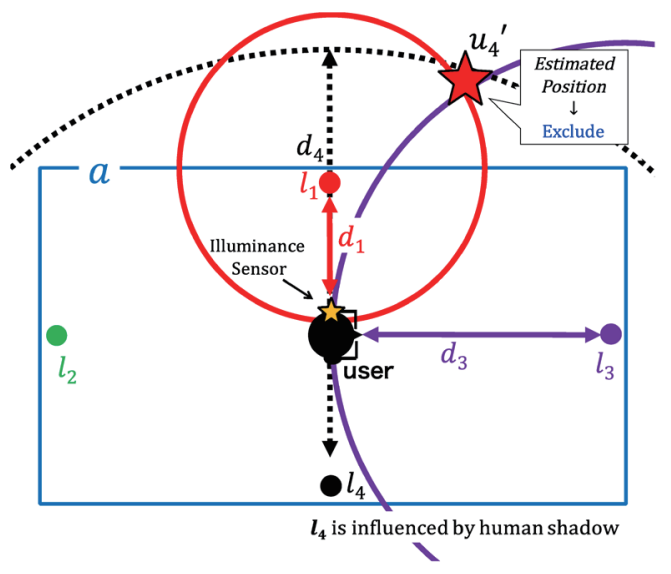

(d)

Fig. 4. (Color online) Example of localization to estimate position of user who is wearing wearable illuminance sensor (case where lighting device $l_{4}$ is influenced by human shadow). (a) Localization utilizing combination pattern $\left\{l_{1}, l_{2}, l_{3}\right\}$. (b) Localization utilizing combination pattern $\left\{l_{1}, l_{2}, l_{4}\right\}$. (c) Localization utilizing combination pattern $\left\{l_{2}, l_{3}, l_{4}\right\}$. (d) Localization utilizing combination pattern $\left\{l_{1}, l_{3}, l_{4}\right\}$. 
positions outside the target area $a$ ) from $U^{\prime}$ as shown in Figs. 4(a)-4(d). The details of this algorithm are described in Algorithm 2 and are summarized as follows:

1. First, the state of all lighting devices used for localization starts from the initial state. The algorithm first obtains the illuminance value in the initial state (lines 1-4).

2. After obtaining the illuminance value in the initial state, the algorithm turns on each lighting device alternately (e.g., $l_{1} \rightarrow l_{2} \rightarrow l_{3} \rightarrow l_{4}$ ) by utilizing the active control. The algorithm calculates the difference between illuminance values obtained before and after turning on a lighting device and obtains the illuminance value after removing the effect of light sources other than the lighting devices used for localization (lines 6-9).

3. The algorithm estimates the distance between the target for measurement and a lighting device by applying the illuminance value obtained by the active control to a distanceilluminance model (line 10).

4. The algorithm repeats procedures 2 and 3 until all distances are calculated (lines 5-11).

5. After calculating each distance, the algorithm calculates each estimated position $U^{\prime}=\left\{u_{1}^{\prime}, u_{2}^{\prime}, u_{3}^{\prime}, u_{4}^{\prime}\right\}$ by utilizing the four combination patterns $\left(\left\{l_{1}, l_{2}, l_{3}\right\},\left\{l_{1}, l_{2}, l_{4}\right\},\left\{l_{2}, l_{3}, l_{4}\right\}\right.$ and $\left.\left\{l_{1}, l_{3}, l_{4}\right\}\right)$ used for localization by the same procedure as Algorithm 1 (line 14).

6. Then, the algorithm excludes all estimated positions that are judged to be outside the target area from the estimated positions $U^{\prime}=\left\{u_{1}^{\prime}, u_{2}^{\prime}, u_{3}^{\prime}, u_{4}^{\prime}\right\}$ to remove the influence of the human shadow (lines 16-19).

7. Finally, by calculating the average estimated position from the remaining estimated positions, the algorithm obtains its position as the final estimated position of the target for measurement (line 20).

\subsection{Distance-illuminance model}

The trilateration-based indoor localization method estimates the position of the target for measurement based on the distance information between the target and at least three anchor nodes whose installation positions are known. Therefore, an effective method that can calculate the distance between them correctly is required to estimate the position of the target for measurement accurately. In this section, we propose a distance-illuminance model as an effective and novel method of calculating the distance between an illuminance sensor and a lighting device to estimate the position of a target for measurement accurately (corresponding to Req. 3). The distance-illuminance model gives the relationship between the distance between the illuminance sensor, which is the target for measurement, and a lighting device, and the illuminance value. The distance-illumination model is created by the following procedure:

1. First, only one lighting device is turned on and the illuminance value is measured at multiple observation points in the target area.

2. Data about the distance between each observation point and the lighting device, as well as illuminance values at these observation points, are recorded.

3. A linear or nonlinear approximation model is then calculated from the recorded data using the least-squares method.

4. Finally, this model is used as the distance-illuminance model. 
The illuminance value obtained by the illuminance sensor gradually decreases as the observation point moves away from the lighting source (illumination device) according to the inverse square law. ${ }^{(31)}$ However, this property varies significantly depending on the measurement environment, the type of illuminance sensor used for measurement, and so on. It is very difficult to create a generic distance-illuminance model that takes into consideration all of these influences. Hence, in this paper, we use a mathematical model created from premeasured illuminance values in the actual target area used for our study as the distanceilluminance model. We discuss preliminary experiments to create a distance-illuminance model in detail in Sect. 5.

\section{Preliminary Experiment}

In this section, we describe the preliminary experiment to create the distance-illuminance models used in 2D and 3D localizations. We first describe the experimental environment and then the method used in the preliminary experiment. Finally, we describe the obtained distance-illuminance models used in 2D and 3D localizations.

\subsection{Experimental environment}

Figure 5 shows the experimental environment in the preliminary experiments. In our study, we assume an ordinary room in a house as the target space/area in the experimental environment. Therefore, we used the smart home in our university, shown in Fig. 5(a), as the testbed to conduct various experiments. Also, the floor size of the target area (colored area), as shown in Fig. 5(b), is $3530 \times 4150 \mathrm{~mm}^{2}$. Since the distance-illuminance model must be created as accurately as possible in our study, preliminary experiments were conducted in the situation of no external light sources (i.e., sunlight, ceiling light, and so forth.) other than the lighting devices used for the measurement. Additionally, to eliminate the effect of furniture and home appliances, they were removed from the target area.

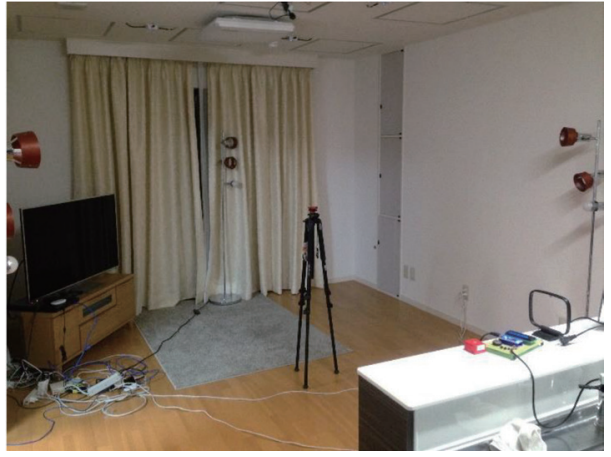

(a)

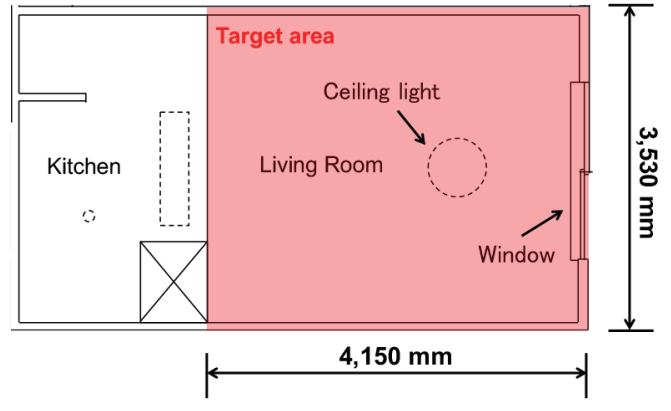

(b)

Fig. 5. (Color online) Experimental environment in preliminary experiment. (a) Smart home testbed. (b) Floor size of target area. 


\subsection{Experimental method}

The major objective of the preliminary experiments was to create the distance-illuminance models used in 2D and 3D localizations according to the procedure described in Sect. 4.3. Also, we consider that investigating the versatility of the proposed method is one of the important objectives of this study. Therefore, we use two different combinations of light devices and illuminance sensors according to each experimental pattern in order to investigate whether the proposed method works effectively even when using different lighting devices. Figure 6 shows the lighting devices (LED light and incandescent light) and the illuminance sensors [SensorTag CC2650STK ${ }^{(23,32)}$ and SenStick ${ }^{(33-36)}$ ] used for the preliminary experiments. We used the combination of the LED light and SensorTag CC2650STK when creating the distanceilluminance model used in 2D localization and the combination of the incandescent light and SenStick when creating the distance-illuminance model used in 3D localization. SenStick is a tiny BLE board in which eight types of sensor are embedded and has been used in various studies on activity recognition and so on. The illuminance sensor mounted on SensorTag CC2650STK is a TEXAS INSTRUMENTS Inc. OPT3001. The illuminance sensor mounted on SenStick is a ROHM Co., Ltd. BH1780GLI.

Figure 7 shows the installation position of the lighting device and the observation points when creating each distance-illuminance model. The observation points used to measure the

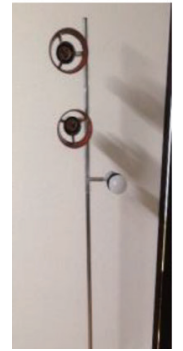

(a)

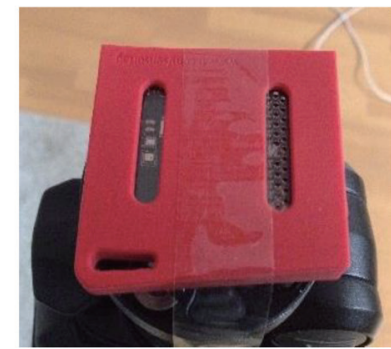

(b)

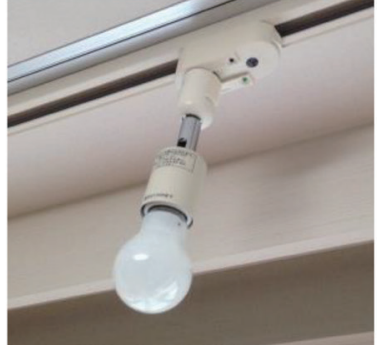

(c)

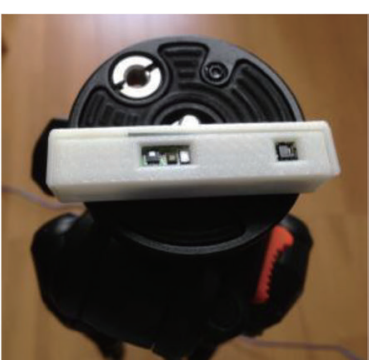

(d)

Fig. 6. (Color online) Devices used in experiment. (a) LED light, (b) SensorTag CC2650STK, (c) incandescent light, and (d) SenStick.

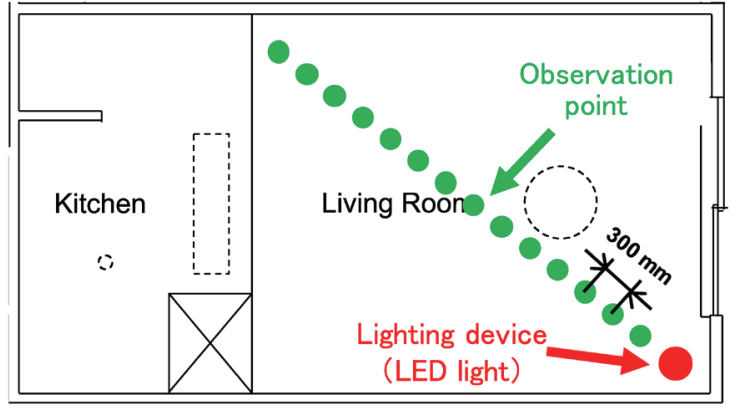

(a)

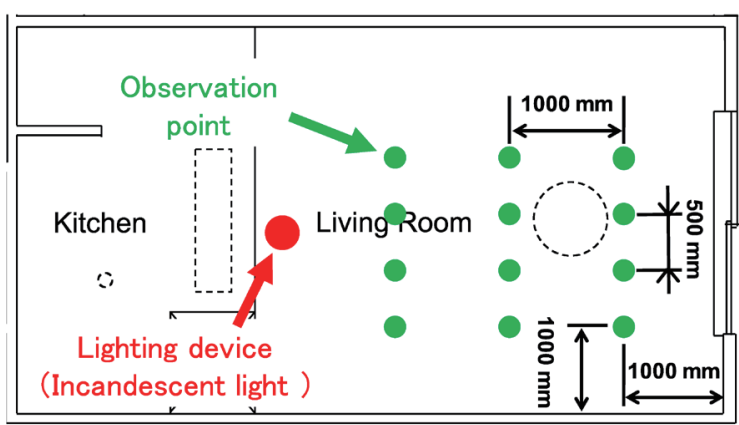

(b)

Fig. 7. (Color online) Installation position of lighting device and observation points. (a) Case of creating distance-illuminance model used for 2D localization. (b) Case of creating distance-illuminance model used for 3D localization. 
illuminance values are set according to the characteristic of the lighting device used in the 2D or 3D localization. To be specific, the observation points shown in Fig. 7(a) are set on a straight line since the LED light (model number: a09099) has high directivity. By contrast, the observation points shown in Fig. 7(b) are set in a grid since the incandescent light (model number: LW100V57W2PA) has high light diffusivity. In the preliminary experiments, we installed the LED light at a height of $1.1 \mathrm{~m}$ and the incandescent light at a height of $2.1 \mathrm{~m}$. Moreover, we measured the illuminance value at each observation point utilizing the illuminance sensor corresponding to each preliminary experiment that installed at a height of $1.1 \mathrm{~m}$.

\subsection{Results of distance-illuminance models used for 2D and 3D localizations}

Figure 8 shows the creation result of each distance-illuminance model. The vertical axis shows the illuminance value and the horizontal axis shows the distance between the illuminance sensor and the lighting device.

From the result in Fig. 8(a), the illuminance value measured at each observation point decreases at almost regular intervals until the distance between the illuminance sensor and the lighting device is $3 \mathrm{~m}$, after which it decreases much more slowly. Therefore, the distanceilluminance model is defined as a function of distance that is piecewise linear. We empirically obtain the approximation model by applying the least-squares method. As a result, the distanceilluminance model for 2D localization is given by

$$
L=\left\{\begin{array}{cc}
-4.21 d+14.96 & (L \geq 3) \\
-0.63 d+5.01 & (L<3)
\end{array}\right.
$$

where $L$ is the illuminance value (lx) and $d$ is the distance between the illuminance sensor and the lighting device (m). On the other hand, from the result in Fig. 8(b), the illuminance value smoothly decreases exponentially as the distance between the illuminance sensor and the lighting device increases. Therefore, we empirically model the data using an approximation

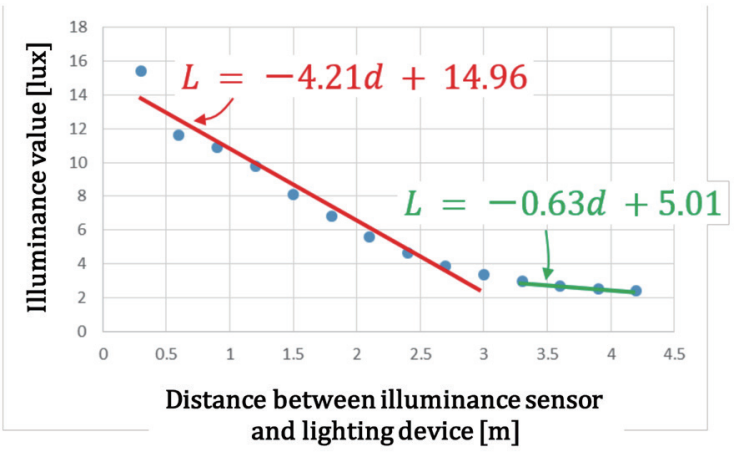

(a)

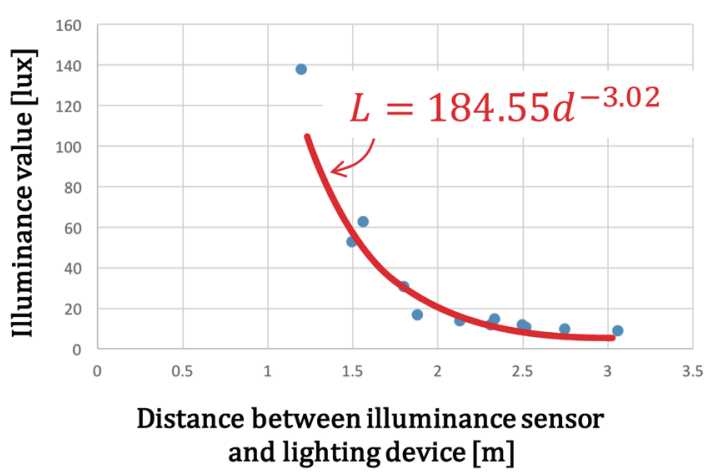

(b)

Fig. 8. (Color online) Creation of distance-illuminance model. (a) Distance-illuminance model used for 2D localization. (b) Distance-illuminance model used for 3D localization. 
curve by applying the least-squares method. As a result, the distance-illuminance model used for $3 \mathrm{D}$ localization is given by

$$
L=184.55 d^{-3.02}
$$

In our study, we conduct localization experiments utilizing these distance-illuminance models. In Sect. 6, we describe evaluation experiments on localization in detail.

\section{Evaluation Experiment}

In this section, we describe the evaluation experiment. We first describe the environment and localization system in the experiments. Then, we describe the evaluation method and discuss the experimental results. Finally, we describe the limitation of the proposed method.

\subsection{Experimental environment}

In this section, we describe the experimental environment. The target area in the evaluation experiments is the same as that in the preliminary experiment described in Sect. 5. The main objective of the evaluation experiment is to determine whether all the requirements mentioned in Sect. 4 are fulfilled. To test the effectiveness of the proposed method in detail, we conduct experiments that assume an actual environment and consider two states: the "ideal state" and "realistic state." In the ideal state, the target space is free from furniture and home appliances, and there are no external light sources (i.e., sunlight or ceiling light) other than the lighting devices used for localization. By contrast, in the realistic state, furniture and home appliances are present and external light sources influence the target space. In this paper, we verify the effectiveness of our proposed method utilizing two experimental patterns (2D and 3D localizations) for each state. Figure 9 shows the layout of the furniture and home appliances in the realistic state used in the 2D and 3D localization experiments. The colored parts show home appliances.

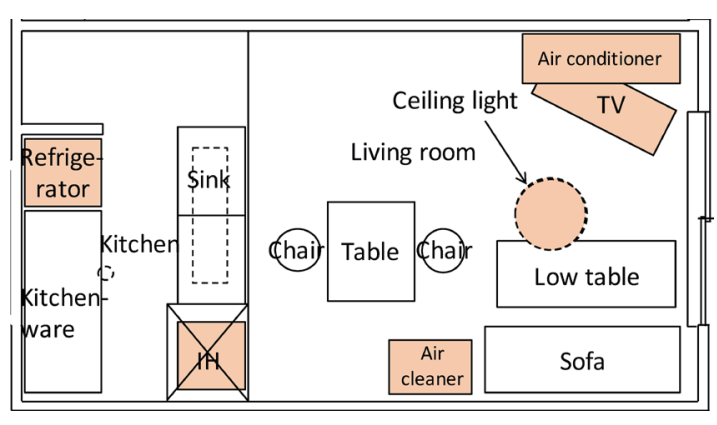

(a)

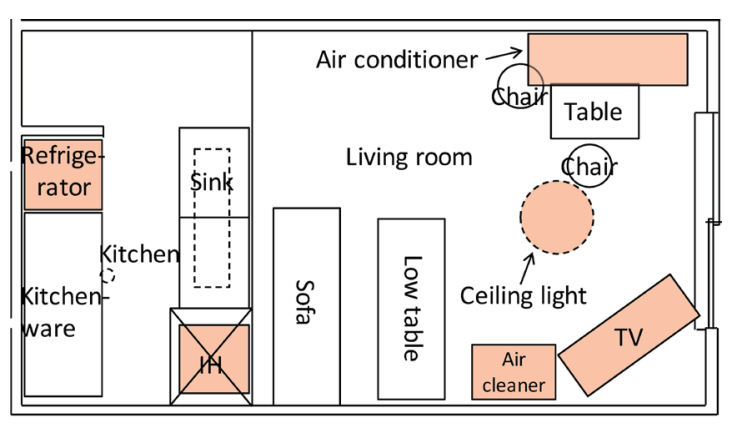

(b)

Fig. 9. (Color online) Layout of furniture and home appliances in realistic state. (a) 2D localization experiment. (b) 3D localization experiment. 


\subsection{Localization system for experiment}

In this section, we describe the localization system for the evaluation experiment. Figure 10 shows the configuration of our localization system. Our system consists of the following five equipment: (1) lighting devices, (2) iRemocon, (3) illuminance sensor, (4) PC, and (5) router. iRemocon is a remote controller controllable via a Wi-Fi network. iRemocon is trained to switch between the state of each lighting device by learning the infrared signal used to control them. Also, iRemocon transmits the control signal to each lighting device when receiving a special character string (control command) by TCP/IP communication. During the localization process, the illuminance sensor (target for measurement), which is installed in the target area, transmits the obtained illuminance values to a PC. The PC controls each lighting device by transmitting the control command (the command to switch the state of each lighting device) to iRemocon via a router. The system estimates the position of the target for measurement by utilizing illuminance values obtained by the illuminance sensor while turning on each lighting device one by one. At this time, the order of switching the state is random.

\subsection{Experimental method}

In this section, we describe the experimental method. We conduct experiments for the following two cases: Case 1) a localization experiment to estimate the position of the illuminance sensor installed in the target area; Case 2) a localization experiment to estimate the position of a user wearing a wearable (glasses-type) illuminance sensor. We describe each case below in detail.

\subsubsection{Localization experiment to estimate position of illuminance sensor (Case 1)}

For Case 1, we estimate the position of the illuminance sensor installed in the target area. We use Algorithm 1, utilizing the active control of three lighting devices in this experiment. In

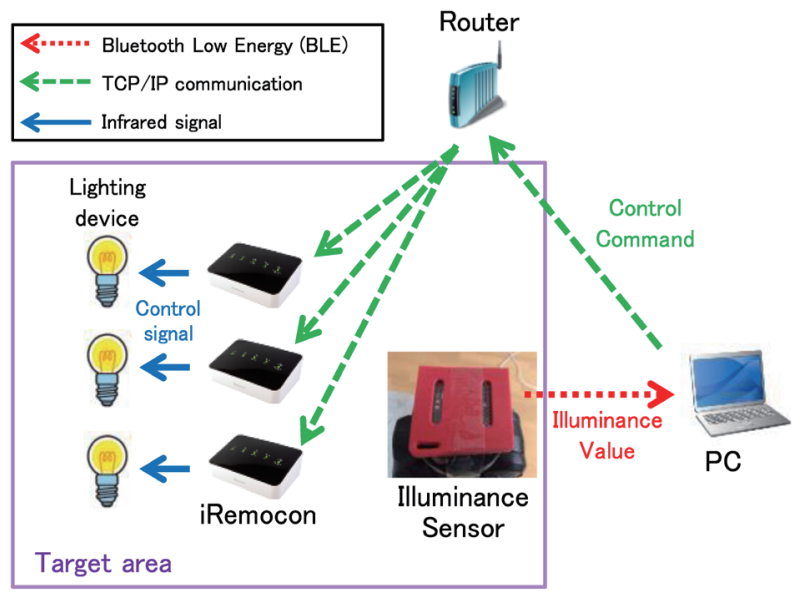

Fig. 10. (Color online) Configuration of localization system for evaluation experiment. 
Case 1, we use two experiment patterns (2D and 3D localizations). Also, the combinations of lighting devices and illuminance sensors used in 2D and 3D localizations are the same as in the previous experiment discussed in Sect. 5. That is, we use the same combination of an LED light and SensorTag CC2650STK in 2D localization and the same combination of an incandescent light and SenStick in 3D localization.

In each experimental pattern in Case 1, we conduct experiments under the following six environmental conditions in Table 1 according to the definitions of the ideal and realistic states: (1) 2D_ideal_state, (2) 2D_with_funiture_appliance (state of ceiling light is OFF, without sunlight, with furniture and home appliances), (3) 2D_with_sunlight (state of ceiling light is OFF, with sunlight, without furniture and home appliances), (4) 2D_ON_ceiling_light (state of ceiling light is $\mathrm{ON}$, without sunlight, furniture, and home appliances), (5) 3D_ideal_state, and 6) 3D_realistic_state. Figures 11(a) and 11(b) show the installation positions of the lighting devices and illuminance sensor in Case 1 for $2 \mathrm{D}$ and 3D localizations, respectively. Small circles show observation points and large circles show the installation positions of the lighting devices. In 2D localization, since the height at which the illuminance sensor is installed must be the same as that of the lighting devices (LED lights), the illuminance sensor is installed at a height of $1.1 \mathrm{~m}$. In 3D localization, to consider the effect of the difference in the height of the installation position of the illuminance sensor, we conduct an experiment utilizing two patterns in which it is installed at heights of 1.0 and $1.5 \mathrm{~m}$. Also, the three incandescent lights used in 3D localization are installed at a height of $2.1 \mathrm{~m}$. The localization process is performed three times at each observation point.

Table 1

Environmental conditions in Case 1.

\begin{tabular}{|c|c|c|c|c|}
\hline Condition & Experimental pattern & $\begin{array}{l}\text { Furniture and home } \\
\text { appliances }\end{array}$ & Sunlight & State of ceiling light \\
\hline 2D_ideal_state & \multirow{4}{*}{ 2D localization } & without & without & OFF \\
\hline $2 \mathrm{D}$ with funiture appliance & & with & without & OFF \\
\hline 2D_with_sunlight & & without & with & OFF \\
\hline 2D_ON_ceiling_light & & without & without & $\mathrm{ON}$ \\
\hline 3D_ideal_state & \multirow{2}{*}{ 3D localization } & without & without & OFF \\
\hline $3 \mathrm{D}$ realistic state & & with & with & $\mathrm{ON}$ \\
\hline
\end{tabular}

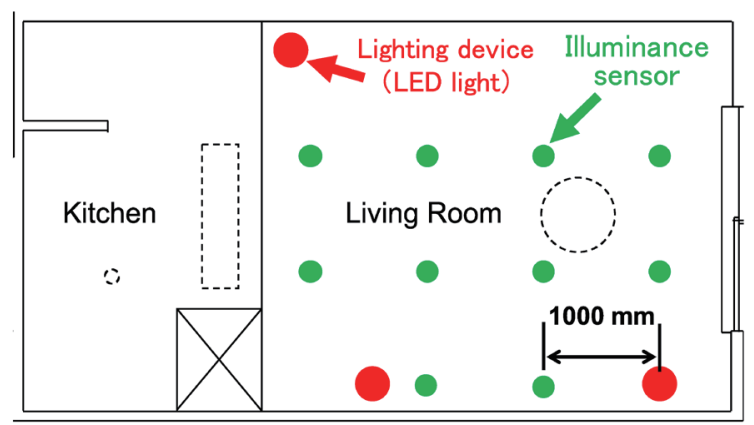

(a)

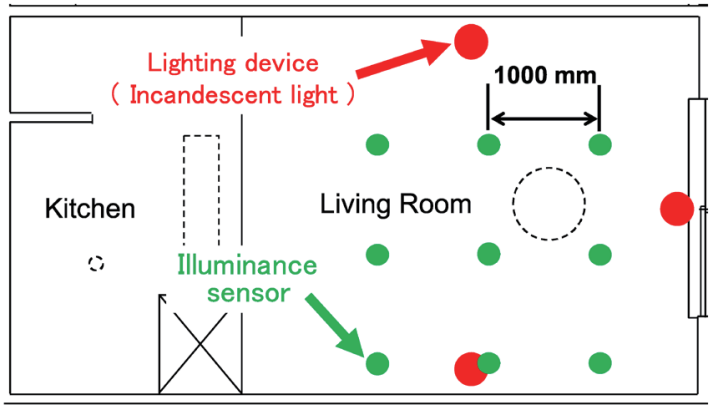

(b)

Fig. 11. (Color online) Installation positions of lighting devices and illuminance sensor in Case 1. (a) 2D localization experiment. (b) 3D localization experiment. 
In Case 1, we evaluate the average localization error (hereafter, called the average error) and the maximum localization error (hereafter, called the maximum error) to show that the proposed method can estimate the position of a target for measurement with high accuracy even with the effect of external light sources, such as sunlight and ceiling light.

\subsubsection{Localization experiment to estimate position of user wearing wearable illuminance sensor (Case 2)}

In Case 2, we estimate the position of a user wearing a wearable illuminance sensor in the target area. We use Algorithm 2, utilizing the active control of four lighting devices in this experiment. We also use Algorithm 1 for comparison to show the superiority of Algorithm 2. The illuminance sensor used in Case 2 is the glasses-type wearable illuminance sensor shown in Fig. 12(a). This wearable illuminance sensor comprises SenStick attached to glasses made by a 3D printer. Figure 12(b) shows the glasses-type illuminance sensor being worn. As shown in Fig. 12(c), during the experiment, the glasses-type illuminance sensor is always worn at a height of $1.5 \mathrm{~m}$, which is the height of the user's eyes.

Case 2 uses the layout shown in Fig. 9(b) and is conducted using only environmental conditions with the realistic state. Figure 13 shows the installation positions of the lighting

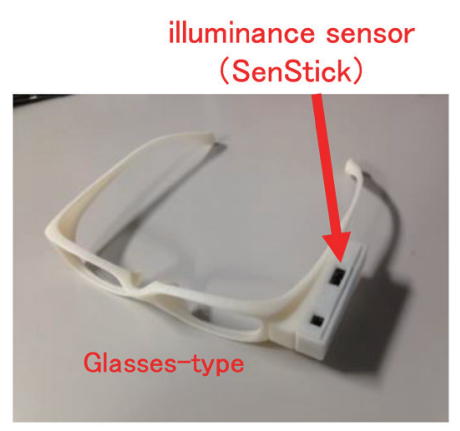

(a)

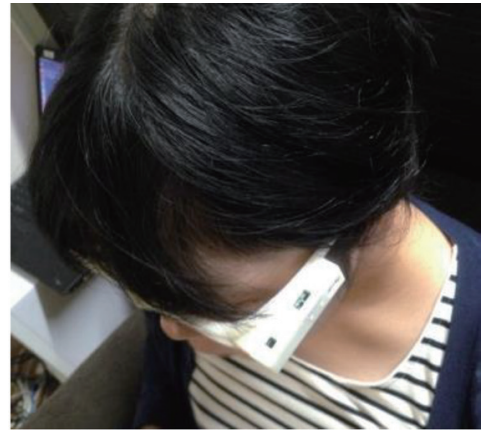

(b)

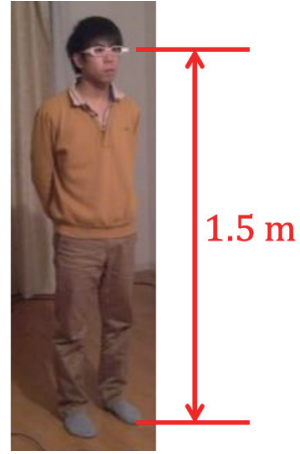

(c)

Fig. 12. (Color online) Wearable illuminance sensor and example of wearing illuminance sensor. (a) Wearable illuminance sensor (glasses-type). (b) Demonstration of glasses-type wearable illuminance sensor. (c) Wearing position during experiment.

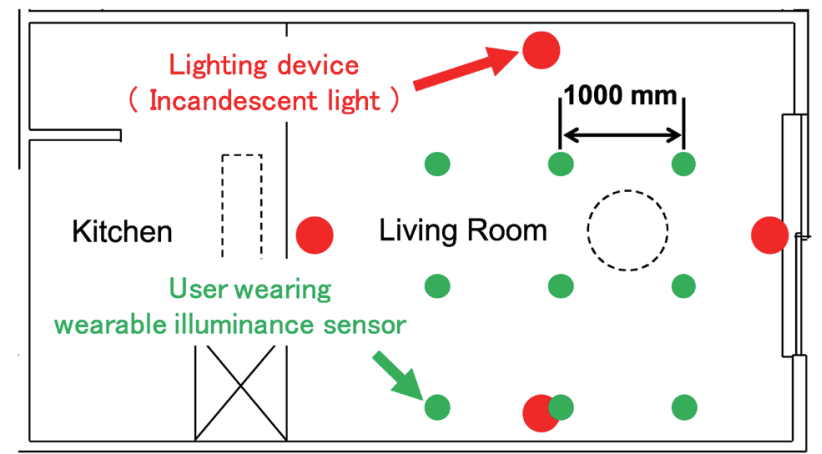

Fig. 13. (Color online) Installation positions of lighting devices and illuminance sensor in Case 2. 
devices and illuminance sensor in Case 2. Small circles show the observation points, where the user stands, and large circles show the installation positions of the lighting devices. In Case 2, we conduct 2D and 3D localizations simultaneously by utilizing the incandescent light installed at a height of $2.1 \mathrm{~m}$. The lighting devices used for Algorithm 1 are the same as in Fig. 11(b). In this experiment, at each observation point, the user faces the direction of the installed lighting devices and performs the localization process. This is done for every lighting device; thus, the localization process is performed 12 times ( $=3$ trials $\times 4$ directions) at each observation point.

In Case 2, we also evaluate the probability that the estimated position is within the target area (called the probability estimate), in addition to the average error and maximum errors, to show that the proposed method can estimate the position of a user wearing an illuminance sensor accurately even in an environment that is influenced by the user's shadow.

\subsection{Results and discussion}

\subsubsection{Experimental results (Case 1)}

Table 2 shows the experimental results in Case 1. The average error (Ave. error) is the average (i.e., 2D: 30 trials $=3$ trials $\times 10$ observation points; 3D: 60 trials $=3$ trials $\times 10$ observation points $\times 2$ patterns) of the localization errors at all observation points. The maximum error (Max. error) is the largest localization error. The difference from the ideal state is the difference between the average errors of the ideal state and the other conditions.

The results in Table 2 show that the average error under all experimental conditions is less than $1 \mathrm{~m}$ and the maximum error is approximately $1 \mathrm{~m}$. Also, the difference from the ideal state under all experimental conditions is less than $0.15 \mathrm{~m}$. These results show that the proposed method can estimate the position of a target for measurement accurately not only in the ideal state but also under experimental conditions assuming the real environment. The proposed method can remove the effect of light sources other than the lighting devices used for localization by applying the difference between illuminance values obtained before and after turning on each lighting device to the appropriate distance-illuminance model according to the experimental pattern. Therefore, the proposed method can estimate the position of a target for measurement with very high accuracy even with the effect of external light sources, such as sunlight and ceiling light. Additionally, from the results in Table 2, we also find that there is almost no change in average error among the different layouts of furniture and home appliances. Hence, we find that the proposed method can fulfill Reqs. 1 and 3 mentioned in Sect. 4.

Table 2

Experimental results in Case 1.

\begin{tabular}{lccc}
\hline Condition & Ave. error $(\mathrm{m})$ & Max. error $(\mathrm{m})$ & Difference from ideal state $(\mathrm{m})$ \\
\hline 2D_ideal_state & 0.44 & 0.85 & - \\
2D_with_funiture_appliance & 0.48 & 1.00 & 0.04 \\
2D_with_sunlight & 0.50 & 1.06 & 0.06 \\
2D_ON_ceiling_light & 0.52 & 1.07 & 0.08 \\
\hline 3D_ideal_state & 0.47 & 0.89 & - \\
3D_realistic_state & 0.61 & 1.13 & 0.14 \\
\hline
\end{tabular}


Figure 14 shows the distribution map of the localization error under each experimental condition. The circles show the size of the average error at each observation point. The results in Fig. 14 show that the average errors of the observation points near the wall are slightly larger than those of other observation points under all experimental conditions. The LED light used in 2D localization has high directivity. Therefore, the average error becomes small at observation points in the measured direction of the distance-illuminance model and becomes slightly larger at observation points near the wall. However, as shown in Figs. 14(a)-14(d), the average error at each observation point is not so large because the average error is less than $1 \mathrm{~m}$ under all experimental conditions. Thus, we consider that the directivity of the LED light has little effect on the localization accuracy. In 3D localization, we consider that the reason why the average error at some observation points including near the wall becomes slightly larger is because the

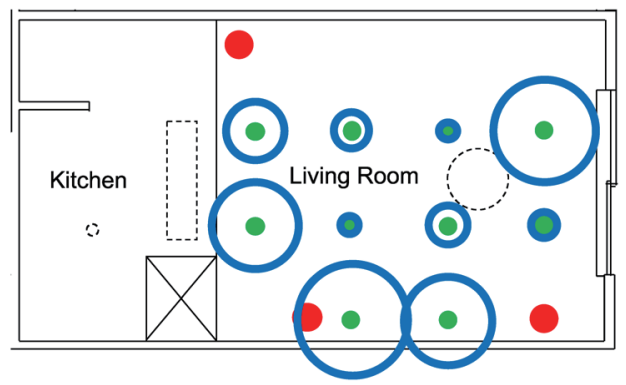

(a)

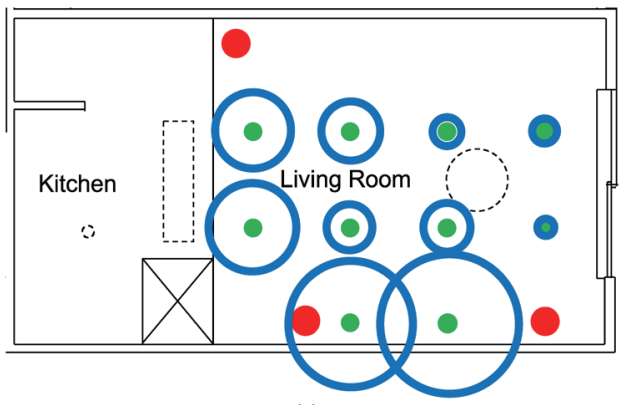

(c)

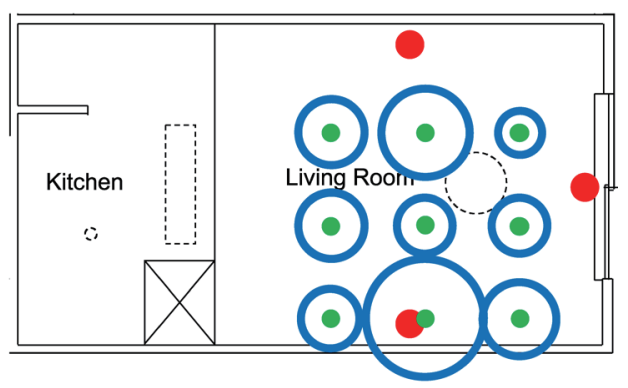

(e)

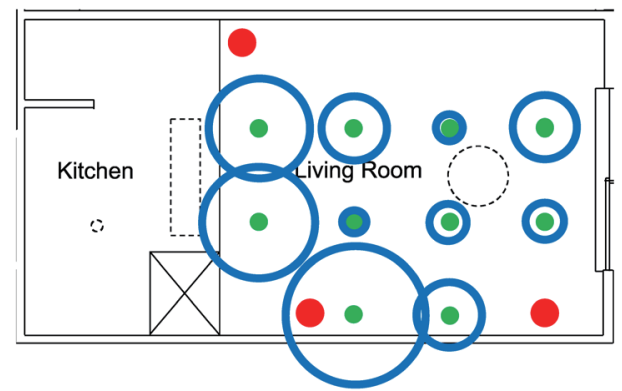

(b)

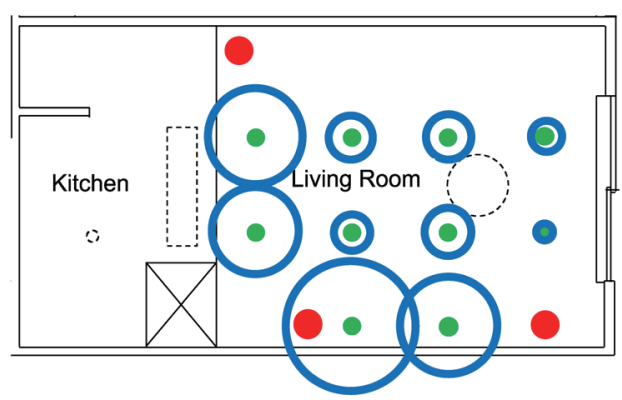

(d)

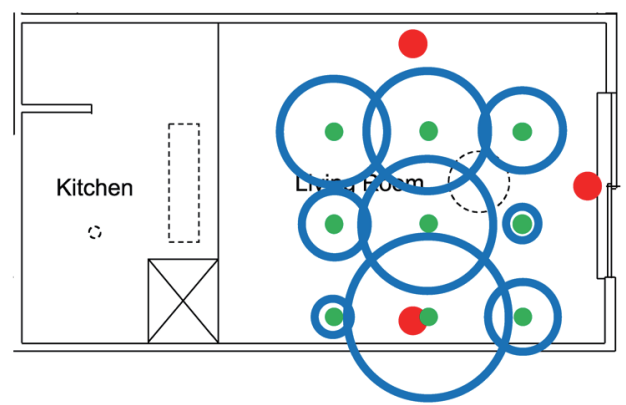

(f)

Fig. 14. (Color online) Distribution map of localization error under each condition. (a) 2D_ideal_state. (b) 2D_ with_funiture_appliance. (c) 2D_with_sunlight. (d) 2D_ON_ceiling_light. (e) 3D_ideal_state. (f) 3D_realistic_state. 
height of the installation position of the illuminance sensor, which is a target for measurement, is different from that in the preliminary experiment. However, as shown in Figs. 14(e) and 14(f), the average error at each observation point is not so large since the average error is less than 1 $m$ under all experimental conditions. Hence, we consider that the difference in the height of the installation position of the illuminance sensor has little effect on the localization accuracy.

\subsubsection{Experimental results (Case 2)}

Table 3 shows the experimental results in Case 2. The average error (Ave. error) is the average (i.e., 108 trials $=3$ trials $\times 4$ directions $\times 9$ observation points) of the localization errors at all observation points. The maximum error (Max. error) is the localization error with the largest value among all localization errors. The probability estimate is the probability that the estimated position of the target for measurement is within the target area. Also, 2D_Alg_1 and 3D_Alg_1 denote that the localization process is performed by Algorithm 1 utilizing the active control of three lighting devices, and 2D_Alg_2 and 3D_Alg_2 denote that it is performed by Algorithm 2 utilizing the active control of four lighting devices.

As shown in Table 3, when the localization process is performed by Algorithm 1, the average errors are 0.96 and $1.17 \mathrm{~m}$ in $2 \mathrm{D}$ and $3 \mathrm{D}$ localizations, respectively. These results show that these conditions have sufficiently high localization accuracy. However, the probability estimate of these conditions is $46.5 \%$, which is relatively low. This result shows that the position of the target for measurement cannot be estimated in more than half of the localization processes. On the other hand, when the localization process is performed by Algorithm 2, the average errors are 0.90 and $1.07 \mathrm{~m}$ in $2 \mathrm{D}$ and $3 \mathrm{D}$ localizations, respectively. These results show that these conditions have sufficiently high localization accuracy. Also, the probability estimate of these conditions is $80.6 \%$, which is higher than that of the conditions utilizing Algorithm 1. This is because Algorithm 2 can remove the influence of a human shadow by effectively utilizing three lighting devices used for localization selected from four lighting devices by the active control. Hence, from these results, we find that the proposed method can fulfill all the requirements mentioned in Sect. 4.

\subsection{Limitation}

Through the experiments in Sect. 6.4, we confirmed the effectiveness of the proposed method. However, our method still has a limitation on the influence of flickering owing to the active control of lighting devices. The influence of flickering caused by the active control of lighting devices changes depending on the frequency of performing the localization process.

Table 3

Experimental results in Case 2.

\begin{tabular}{lccc}
\hline Condition & Ave. error $(\mathrm{m})$ & Max. error $(\mathrm{m})$ & Probability estimate (\%) \\
\hline 2D_Alg_1 & 0.96 & 2.76 & 46.5 \\
2D_Alg_2 & 0.90 & 1.73 & 80.6 \\
3D_Alg_1 & 1.17 & 2.76 & 46.5 \\
3D_Alg_2 & 1.07 & 2.49 & 80.6 \\
\hline
\end{tabular}


If we track a user's position frequently, the proposed method needs to continuously perform active control. As a result, the user might feel discomfort. By contrast, we consider that the user will not feel flickering when the proposed method is used periodically at fixed intervals (e.g., approximately once every 10 or $30 \mathrm{~min}$ ). Also, we consider that it is likely that a user feels discomfort when the active control of lighting devices is performed during a specific activity, e.g., watching TV. We consider that the discomfort to the user may be reduced by performing the active control of lighting devices while avoiding the specific activity recognized by certain methods. ${ }^{(8,9)}$

\section{Conclusion}

In this paper, we have proposed a trilateration-based indoor localization method utilizing active control of lighting devices. The proposed method estimates the position of the target for measurement by utilizing a trilateration method based on the distances between the target for measurement and lighting devices calculated from the difference in illuminance value obtained by the active control. To show the effectiveness of the proposed method, we conducted evaluation experiments for two cases: (1) a localization experiment to estimate the position of the illuminance sensor installed in the target area and (2) a localization experiment to estimate the position of a user wearing a wearable illuminance sensor. The following are primary findings of this study:

- The proposed method can estimate the target's position with $1 \mathrm{~m}$ accuracy or higher even with the influence of external light sources (such as sunlight and ceiling light) other than the lighting devices used for localization by considering only the difference between illuminance values obtained before and after turning on each lighting device.

- The proposed method can estimate the target's position with approximately $1 \mathrm{~m}$ accuracy even in an environment influenced by a human shadow by effectively utilizing three lighting devices selected from four lighting devices by the active control.

- The proposed method can estimate the target's position accurately by utilizing the appropriate distance-illumination model according to each experimental pattern, not only in the ideal state but also under experimental conditions simulating a real environment.

As future work, we plan to optimize how to select two circles/spheres in our algorithm, e.g., we consider that we need to try all combination patterns and then select the best combination pattern among them. Also, we will devise a distance-illuminance model that takes into account the influence of light reflection by a wall. Even if multiple targets for measurement exist in the target area, we will devise a localization algorithm that can estimate the position with high accuracy. Additionally, we will devise an optimal placement method for lighting devices used for localization.

\section{Acknowledgments}

This work was partly supported by JSPS KAKENHI Grant Numbers JP16H01721, JP16K00126, and JP19H05665. 


\section{References}

1 J. Paek, J. G. Ko, and H. Shin: Mobile Inf. Syst. 2016 (2016) 1. https://doi.org/10.1155/2016/8367638

2 D. Chen, K. G. Shin, Y. Jiang, and K.-H. Kim: Proc. 13th Int. Conf. Emerging Networking Experiments and Technologies (ACM, 2017) 263. http://doi.acm.org/10.1145/3143361.3143385

3 J. Scott, A. J. B. Brush, J. Krumm, B. Meyers, M. Hazas, S. Hodges, and N. Villar: Proc. 13th Int. Conf. Ubiquitous Computing (ACM, 2011) 281. http://doi.acm.org/10.1145/2030112.2030151

4 S. Barker, A. Mishra, D. Irwin, P. Shenoy, and J. Albrecht: Proc. 2012 IEEE Int. Conf. Pervasive Computing and Communications (IEEE, 2012) 67. https://doi.org/10.1109/PerCom.2012.6199851

5 K.-H. Park, Z. Bien, J.-J. Lee, B. K. Kim, J.-T. Lim, J.-O. Kim, H. Lee, D. H. Stefanov, D.-J. Kim, J.-W. Jung, J.-H. Do, K.-H. Seo, C. H. Kim, W.-G. Song, and W.-J. Lee: Auton. Rob. 22 (2007) 183. https://doi.org/10.1007/ s10514-006-9012-9

6 S. Inoue, N. Ueda, Y. Nohara, and N. Nakashima: Proc. 2015 ACM Int. Joint Conf. Pervasive and Ubiquitous Computing (ACM, 2015) 1269. http://doi.acm.org/10.1145/2750858.2807533

7 T. Morita, K. Taki, M. Fujimoto, H. Suwa, Y. Arakawa, and K. Yasumoto: J. Sens. 2018 (2018) 1. https://doi. org/10.1155/2018/2625195

8 K. Moriya, E. Nakagawa, M. Fujimoto, H. Suwa, Y. Arakawa, A. Kimura, S. Miki, and K. Yasumoto: Proc. 1st Int. Workshop on Mobile and Pervasive Internet of Things (IEEE, 2017) 1. https://doi.org/10.1109/ PERCOMW.2017.7917603

9 E. Nakagawa, K. Moriya, H. Suwa, M. Fujimoto, Y. Arakawa, and K. Yasumoto: Proc. 1st Int. Workshop on Pervasive Smart Living Spaces (IEEE, 2017) 1. https://doi.org/10.1109/PERCOMW.2017.7917620

10 K. Ueda, H. Suwa, Y. Arakawa, and K. Yasumoto: Proc. 14th IEEE Int. Conf. Ubiquitous Computing and Communications (IEEE, 2015) 1130. https://doi.org/10.1109/CIT/IUCC/DASC/PICOM.2015.169

11 J. Krumm, S. Harris, B. Meyers, B. Brumitt, M. Hale, and S. Shafer: Proc. 3rd IEEE Int. Workshop on Visual Surveillance (IEEE, 2000) 1. https://doi.org/10.1109/VS.2000.856852

12 N. Checka, K. Wilson, V. Rangarajan, and T. Darrell: Proc. 2003 Conf. Computer Vision and Pattern Recognition Workshop (IEEE, 2003) 1. https://doi.org/10.1109/CVPRW.2003.10099

13 D. Hähnel, W. Burgard, D. Fox, K. Fishkin, and M. Philipose: Proc. 2004 IEEE Int. Conf. Robotics \& Automation (IEEE, 2004) 1015. https://doi.org/10.1109/ROBOT.2004.1307283

14 M. Fujimoto, T. Wada, A. Inada, K. Mutsuura, and H. Okada: IEICE Trans. Fundam. E95-A (2012) 1596. https://doi.org/10.1587/transfun.E95.A.1596

15 V. C. Paterna, A. C. Augé, J. P. Aspas, and M. A. P. Bullones: Sens. 2017 (2017) 1. https://www.mdpi. com/1424-8220/17/12/2927

16 X. Tian, W. Li, Y. Yang, Z. Zhang, and X. Wang: IEEE Trans. Mob. Comput. 17 (2018) 390. https://doi. org/10.1109/TMC.2017.2715820

17 Z. Yang, C. Wu, and Y. Liu: Proc. 18th Annual Int. Conf. Mobile Computing and Networking (ACM, 2012) 269. http://doi.acm.org/10.1145/2348543.2348578

18 L. Wang, T.-K. Hon, J. D. Reiss, and A. Cavallaro: IEEE Trans. Signal Process. 64 (2016) 1018. https://doi. org/10.1109/TSP.2015.2498130

19 S. A. Golden and S. S. Bateman: IEEE Trans. Mob. Comput. 6 (2007) 1185. https://doi.org/10.1109/ TMC.2007.1002

20 D. Niculescu and B. Nath: Proc. 22nd Annual Joint Conf. the IEEE Computer and Communications Societies (IEEE, 2003) 1734. https://doi.org/10.1109/INFCOM.2003.1209196

21 W. Zhang, M. I. S. Chowdhury, and M. Kavehrad: Opt. Eng. 53 (2014) 1. https://doi.org/10.1117/1. OE.53.4.045105

22 K. Yamaguchi, M. Miki, S. Kuwajima, R. Jonan, and H. Aida: Proc. 2015 Int. Conf. Artificial Intelligence (ICAI, 2015) 503.

23 K. Moriya, M. Fujimoto, Y. Arakawa, and K. Yasumoto: Proc. 7th Int. Conf. Indoor Positioning and Indoor Navigation (IEEE, 2016) 1. https://doi.org/10.1109/IPIN.2016.7743623

24 C. Xiao, D. Yang, Z. Chen, and G. Tan: IEEE Access 5 (2017) 12751. https://doi.org/10.1109/ ACCESS.2017.2720164

25 F. Zafari, A. Gkelias, and K. K. Leung: IEEE Commun. Surv. Tutor. 21 (2019) 2568. https://doi.org/10.1109/ COMST.2019.2911558

26 A. R. Pratama, Widyawan, and R. Hidayat: Proc. 2012 Int. Conf. System Engineering and Technology (IEEE, 2012) 1. https://doi.org/10.1109/ICSEngT.2012.6339316

27 Active Bat: http://www.cl.cam.ac.uk/research/dtg/attarchive/bat/ (Accessed: Sept. 2019) 
28 J. Xiao, K. Wu, Y. Yi, L. Wang, and L. M. Ni: Proc. IEEE 33rd Int. Conf. Distributed Computing Systems (IEEE, 2013) 236. https://doi.org/10.1109/ICDCS.2013.49

29 Y. Kashimoto, M. Fujimoto, H. Suwa, Y. Arakawa, and K. Yasumoto: Proc. 7th International Conference on Indoor Positioning and Indoor Navigation (IEEE, 2016) 1. https://doi.org/10.1109/IPIN.2016.7743667

30 C. Jiang, Y. He, S. Yang, J. Guo, and Y. Liu: Proc. 18th Int. Conf. Information Processing in Sensor Networks (ACM, 2019) 25. http://doi.acm.org/10.1145/3302506.3310386

31 M. Liao, L. Wang, R. Yang, and M. Gong: Proc. 2007 IEEE Conf. Computer Vision and Pattern Recognition (IEEE, 2007) 1. https://doi.org/10.1109/CVPR.2007.383159

32 TEXAS INSTRUMENTS Inc.: http://www.ti.com/tool/CC2650STK (Accessed: Sept. 2019).

33 Y. Nakamura, Y. Arakawa, T. Kanehira, M. Fujiwara, and K. Yasumoto: J. Sens. 2017 (2017) 1. https://doi. org/10.1155/2017/6308302

34 M. Takata, Y. Nakamura, M. Fujimoto, Y. Arakawa, and K. Yasumoto: Proc. 2018 ACM Int. Joint Conf. 2018 Int. Symp. Pervasive and Ubiquitous Computing and Wearable Computers (ACM, 2018) 1404. https://doi. org $/ 10.1145 / 3267305.3267504$

35 Y. Matsuda, D. Fedotov, Y. Takahashi, Y. Arakawa, K. Yasumoto, and W. Minker: Sens. 18 (2018) 1. https:// doi.org/10.3390/s18113978

36 Y. Umetsu, Y. Nakamura, Y. Arakawa, M. Fujimoto, and H. Suwa: Proc. 2019 IEEE Int. Conf. Pervasive Computing and Communications (IEEE, 2019) 222. https://doi.org/10.1109/PERCOM.2019.8767385 